\title{
Une étude synthétique des phénomènes d'adaptabilité en période de crise
}

Les pêcheurs morbihannais face à la crise sardinière (1881-1913)

\section{Xavier Dubois}

\section{(2) OpenEdition}

\section{Journals}

Édition électronique

URL : http://journals.openedition.org/abpo/1628

DOI : $10.4000 / a b p o .1628$

ISBN : 978-2-7535-1485-0

ISSN : 2108-6443

Éditeur

Presses universitaires de Rennes

Édition imprimée

Date de publication : 20 mars 2002

Pagination : 91-106

ISBN : 978-2-86847-708-8

ISSN : 0399-0826

Référence électronique

Xavier Dubois, "Une étude synthétique des phénomènes d'adaptabilité en période de crise », Annales de Bretagne et des Pays de l'Ouest [En ligne], 109-1 | 2002, mis en ligne le 20 juin 2004, consulté le 01 mai 2019. URL : http://journals.openedition.org/abpo/1628 ; DOI : 10.4000/abpo.1628 


\title{
Une étude synthétique des phénomènes d'adaptabilité en période de crise
}

\author{
Les pêcheurs morbihannais \\ face à la crise sardinière (1881-1913)
}

\author{
Xavier DuBoIs \\ Doctorant, laboratoire du SoLiTo, Université de Bretagne-Sud
}

Conséquence de la mise en application de la technique de conservation inventée par Nicolas Appert en 1804, l'implantation de conserveries sur le littoral breton va entraîner un développement rapide des pêches sardinières, selon un processus encore mal étudié. Les sardines vont en effet constituer un des produits les mieux adaptés à ce procédé. Dès les années 1830-1840, le succès grandissant de ce nouveau type de produit - dont les qualités gustatives sont préservées, et dont la durée de conservation surpasse celle des procédés traditionnels (presse, salage, séchage) - détermine l'apparition d'un nouveau marché commercial, dont les limites dépassent les frontières nationales. La sardine ainsi conservée va rapidement devenir un produit essentiellement destiné à l'exportation. Ce nouveau type d'activité, qui remplace la pratique traditionnelle de la presse, va engendrer au niveau local et régional de profondes mutations. Les communes littorales bénéficient de ce fait d'un processus d'industrialisation jusqu'alors inédit en Bretagne. Les ports sardiniers connaissent un fort développement démographique en même temps que de profondes mutations sociales. Les rapports humains et socio-économiques sont profondément bouleversés par ce passage d'une économie traditionnelle à caractère régional à une économie de marché de niveau international. Cette transition suscite également diverses évolutions technologiques, bien que la plus grande partie des matériels (chaloupes) et méthodes de pêche (pêche au filet droit) soient encore directement issue des techniques employées au siècle précédent.

À partir des années 1880, l'industrie sardinière bretonne, qui détient jusqu'alors une position incontestée de leader au niveau mondial, est brutalement confrontée à une crise de production. Du fait de variations climatiques, les bancs se sont raréfiés et la pêche enregistre de très médiocres résultats. Alors que les premières années du $\mathrm{XX}^{\mathrm{e}}$ siècle laissent augurer un retour à 
une situation plus stable, une autre crise apparaît à partir de $1902^{1}$. La disparition ponctuelle des bancs se conjugue alors avec la crise des débouchés. Les pays concurrents de la France, qui ont profité de la première crise sardinière pour rattraper leur retard technologique, se sont en effet placés sur le marché international mettant la Bretagne dans une situation délicate à laquelle les acteurs de l'économie sardinière n'étaient pas préparés ${ }^{2}$.

Pour déterminer quelles étaient les capacités d'adaptabilité des acteurs sardiniers aux crises, et pour comprendre pourquoi ceux-ci pouvaient au contraire demeurer figés dans une situation relativement immobiliste, nous avons travaillé sur les archives de la Marine conservées à Lorient. Les registres de rôles de bord au désarmement dans les quartiers du Morbihan entre 1900 et $1910^{3}$, période qui nous semble la plus représentative au niveau de l'évolution des armements, ont constitué notre principale source. Si nous avons fait ce choix de restreindre le cadre géographique de notre étude à la Bretagne Sud, c'est parce qu'il existe des différences notables dans l'organisation socio-économique entre cette région et les départements voisins. Dans l'espace sardinier breton, il existe une spécificité morbihannaise. Toutefois, l'industrie sardinière de Bretagne Sud subit l'influence financière et syndicale de Nantes, Douarnenez et Concarneau.

\section{Une modification des structures professionnelles de l'armement sardinier}

Une des principales conséquences de la crise sardinière est, faut-il le préciser, une diminution notable des revenus des acteurs des systèmes sardiniers (pêcheurs, armateurs, conserveurs...). Aussi les armateurs, bien moins dépendants de cette ressource que les pêcheurs - assez souvent spécialisés dans cette quasi monoactivité que représente cette pêche sardinière vont-ils, dès les années 1880, se désengager massivement de cette activité, devenue source de pertes, au profit d'autres investissements ou d'autres types de pêche. Les sommes investies, en effet, dépassent trop souvent en période de crise la valeur même des prises. Et lors des périodes de reprise (ou plus exactement de répit) économique, les bénéfices récoltés sont beaucoup plus limités que durant la période antérieure aux crises. Aussi les armateurs se tournent-ils vers la pêche du thon, celle du poisson frais, voire de la morue verte, dont les gains sont plus réguliers. Ils s'orientent également vers le chalutage. Cette activité profite de reports d'investissement des capi-

1. Avigneau, André-Marie d', L'industrie des conserves de poisson en France métropolitaine. Analyse économique du développement de la branche et diagnostic des entreprises. Thèse de doctorat de sciences économiques, Université de Rennes, 1958, dactyl.

2. DuBoIs, Xavier, "La mondialisation en Bretagne à travers la mutation des systèmes sardiniers (milieu XIX ${ }^{\mathrm{e}}$-début Xx $\mathrm{x}^{\mathrm{e}}$ siècle) ", La Bretagne à l'heure de la mondialisation, premières journées de la recherche en sciences humaines et sociales de Bretagne, Rennes 2000 (à paraître : Presses Universitaires de Rennes, 2002).

3. Archives du service historique de la Marine (SHM), Lorient, séries 3 P, 6 P, 8 P et 9 P, Registres des rôles de bord au désarmement. 
taux sardiniers, et le port de Lorient sera le grand bénéficiaire de cette nouvelle orientation économique. Dès l'extrême fin du XIX ${ }^{\mathrm{e}}$ siècle cependant, les possibilités de reconversion des petits armateurs ou patrons-armateurs dans le chalutage sont paradoxalement limitées par l'extension de cette activité. Ceux-ci doivent en effet soutenir une lutte de plus en plus âpre contre les gros armateurs qui s'approprient le marché. L'intrusion de la vapeur et de l'ottertrawl ${ }^{4}$ ne fera qu'aggraver cette situation, en avilissant les cours, en raison des volumes introduits sur le marché. Les prises des petits chalutiers traditionnels sont de ce fait moins rémunératrices. Les patrons sardiniers hésitent donc à investir des sommes importantes en vue d'une reconversion, qui pourrait sembler aléatoire, dans le chalutage. Les armateurs de Quiberon préfèrent donc suivre l'exemple camarétois et se lancent dans la pêche aux crustacés, opérant cette diversification en munissant de viviers une partie de leurs caboteurs, ou en acquérant des dundees ainsi équipés. Les patrons de Quiberon, Saint-Pierre, Sauzon, Lomener ou Groix se dotent également de petits caseyeurs, canots ou petits sloops demi-pontés. La crise sardinière confirme les pêcheurs de Groix dans leur orientation thonière, espèce dont la pêche est moins aléatoire que celle de la sardine et offre de meilleurs revenus. D'autres patrons reconvertissent leur navire de pêche en caboteur, spécialement s'il s'agit d'une forte chaloupe, après lui avoir fait subir quelques modifications. Au commencement des années 1910, une dizaine d'équipages morbihannais, essentiellement grésillons, se tournent vers le trafic de sardines en contrebande. Sous couvert de pêche sardinière ou langoustière dans les eaux portugaises ou nord-africaines, les marins achètent la sardine aux pêcheurs locaux, au Maroc ou au Portugal, et la transportent en demi-sel à destination des marchés français. Mais ce type de diversification interlope reste marginal.

Mais si ces reconversions dépendent des capacités d'évolution des modes de financement et d'armement, les possibilités de débouchés doivent être également prises en compte. L'attrait des activités germonières, par exemple, ne sera possible que dans la mesure où les infrastructures portuaires seront appropriées aux types d'unités employées et où le potentiel industriel sera suffisamment étoffé. Les capacités de reconversion sont également influencées dans une large mesure par l'état d'esprit des pêcheurs sardiniers (corporatisme, conservatisme) ${ }^{5}$. Ainsi, ces pêcheurs semblent réticents à s'impliquer dans les activités de chalutage, malgré la crise et des salaires attractifs, comme semble l'indiquer cette note de 1894 : "En raison du manque de main d'œuvre, les patrons ont du mal à former leurs équipages. Il n'existe pas de chômage dans cette pêche ${ }^{6}$. "

4. Ottertrawl : type de chalut à panneaux, qui remplace peu à peu le traditionnel chalut à perche.

5. Dubols, Xavier, "Mutations techniques et crises sardinières ", Mutations techniques des pêches maritimes : agir ou subir? Évolution des systèmes techniques et sociaux, colloque de Lorient, 1999, à paraître aux éditions IFREMER (sous presse).

6. Arch. dép. du Morbihan, 9 M 3, Situations industrielles, deuxième semestre 1894 . 
Ainsi, en raison de ce désengagement, la proportion d'armateurs sardiniers extérieurs (c'est à dire qui ne sont pas eux-mêmes patrons) diminue-t-elle considérablement et rapidement. À Belle-Île, elle passe de 66,7 à $19,3 \%$ des armateurs sardiniers entre 1868 et 1900, puis diminue encore pour atteindre $7,8 \%$ en $1910^{7}$. Cette évolution est sensiblement identique dans l'ensemble des quartiers maritimes de l'arrondissement. En conséquence, les patrons de pêche sont contraints d'assumer eux-mêmes les armements. La proportion des patrons armateurs atteint-elle ainsi dans les années 1900 à 1913, 70 \%, 80 \% et même jusqu'à 90 \% des armateurs dans certains ports $^{8}$. Afin d'assumer cette charge financière supplémentaire, les patrons s'orientent vers la solution du co-armement (10 à $12 \%$ des armements sardiniers en moyenne), mais ont également recours à l'aide financière de leur cellule familiale, qui peut assurer dans les cas extrêmes jusqu'à $20 \%$ des armements. Enfin, une autre solution consiste à solliciter une personne de confiance qui va investir dans l'armement de manière occasionnelle. Il s'agit fréquemment d'un patron de pêche retraité (demi-soldier) qui assume ces frais jusqu'à ce que le marin qui a fait appel à lui ait recueilli suffisamment de gains pour racheter son embarcation.

Parallèlement à ces évolutions structurelles des modes de financement de l'armement, les caisses de Crédit Maritime vont se développer sur le littoral. Le développement de ces organismes doit beaucoup à l'impulsion de l'État, de certains députés locaux, mais aussi de certains syndicats d'inscrits maritimes. Un des objectifs de ces syndicats, qui s'étaient rapidement développés sur l'ensemble du littoral atlantique dès 1881, consistait à institutionnaliser une solidarité professionnelle déjà effective dans le but de faire face aux difficultés liées à la crise. Les pêcheurs morbihannais démontrent donc qu'ils sont capables de faire preuve d'une capacité d'organisation et de regroupement. Cependant, l'initiative syndicale revient en premier lieu à des centres sardiniers situés dans d'autres départements, tels ceux de Douarnenez ou Concarneau, où émerge une forte activité revendicative. Les structures syndicales morbihannaises sont également dépendantes des fédérations syndicales nationales, et ne font souvent que prendre le relais de décisions prises au niveau de ces fédérations. Les capacités d'initiative et les pouvoirs décisionnaires locaux sont donc limités, hormis dans certains cas de conflits locaux aux caractéristiques spécifiques.

Les objectifs de ces regroupements ne se limitent pas aux aspects revendicatifs et corporatifs. En effet, les structures syndicales jouent également un rôle coopératif, en complément du Crédit Maritime. Les achats groupés effectués par l'intermédiaire de ces associations permettent de réduire parfois de moitié le prix de revient des matériels de pêche (engins ou embarcation). Cependant certains patrons-armateurs, comme ceux de Belle-lle ou de Concarneau, préfèrent avoir recours au système du prêt direct consenti avec hypothèque sur le bateau.

7. SHM Lorient, série $3 \mathrm{P} 2$, registres de rôles de bord au désarmement.

8. SHM Lorient, séries 3 P 2, 6 P 4, 6 P 6, 8 P 1, 9 P 1 et 9 P 4. 


\section{La reconversion des équipages sardiniers et l'évolution des méthodes de travail}

\section{Une modification de la politique de recrutement des équipages}

Les patrons réduisent autant que possible leurs équipages, entraînant une baisse générale des effectifs des flottilles. À ces hésitations devant l'embauche s'ajoute une sélection plus rigoureuse des matelots. Cette sélection, conjuguée à la disproportion qui existe désormais entre l'offre et la demande, entraîne une baisse notable de la proportion des non inscrits réguliers (agriculteurs, salariés agricoles) parmi les effectifs. La pratique du recrutement d'équipage d'origine rurale était en effet jusqu'alors importante dans certaines localités où la faible densité de population "maritime " limitait la réserve de main d'œuvre disponible, comme dans les ports de Belle-Île ou à Port-Louis. Dans ce dernier port par exemple, les paysans composaient encore à la veille de la crise (en 1878) en moyenne $40 \%$ des équipages ${ }^{9}$. Cette proportion diminuera considérablement à partir des années 1880. Les familles rurales qui avaient coutume de s'engager pour la saison perdent ainsi une source de revenu complémentaire et non négligeable.

Certains matelots choisissent donc la voie de la reconversion dans d'autres activités halieutiques comme celle de la pêche du thon ${ }^{10}$. D'autres se rabattent sur le chalutage des espèces de fond (drague) pour assurer un approvisionnement en poisson frais. Mais bien qu'il connaisse une forte expansion du fait du développement des techniques et des réseaux de transports, ce marché reste limité et fortement concurrencé. La surpêche qui en résulte entraîne donc sur certains sites un appauvrissement des espèces de fond. En outre, les pêcheurs sont confrontés à des problèmes d'inadéquation de leur matériel (en particulier les embarcations) face au type de pêche effectué. D'autres enfin, moins nombreux s'embarquent dans les grands ports français (en particulier à Nantes et Bordeaux) à bord des vapeurs ou des derniers grands voiliers de commerce. Certains constituent une partie des équipages des premiers chalutiers à vapeur lorientais ou rochelais. Il semble cependant que la majorité des marins restent fidèles à la pêche sardinière, conservant l'espoir d'un retour à un certain "âge d'or " ayant précédé la crise ${ }^{11}$.

Cette réduction de la proportion des matelots parmi les équipages ne modifie qu'assez peu l'esprit corporatiste qui règne au sein de ce milieu

9. SHM Lorient, 9 P 1/8-9, Registre des rôles de bord au désarmement, sous-quartier de Lorient, 1878; 9 P 3/37, Registre de la matricule des inscrits définitifs, syndicat de PortLouis, 1878.

10. Une partie du recrutement des équipages thoniers d'Étel, mais surtout de Groix, s'effectue au sein d'autres quartiers, en particulier ceux voisins de Belle-lle, Auray, Lorient, Concarneau et Douarnenez.

11. À ce sujet, les travaux de recherche actuellement en cours au sein du laboratoire d'Histoire et Sciences Sociales sur le Littoral et la Mer (SoLITO) apporteront sans doute d'utiles éclaircissements. 
professionnel et qui entraîne une véritable cohésion entre matelots et patrons, à la seule exception d'un litige local qui éclate à Sauzon en juin $1913^{12}$. Celui-ci oppose marins-pêcheurs et patrons au sujet de la répartition traditionnelle des parts. Si ce conflit est marginal et se dénoue rapidement, il dénote tout de même clairement une certaine dégradation des rapports professionnels après plusieurs années de crise. Ainsi, les années 1900-1910 verront la naissance de certains syndicats distincts de marins et de patrons, bien que, toutefois, la majorité de ces syndicats reste mixte. Suivant cette même logique, la proportion de mousses parmi les équipages de certains ports diminue également, d'autant que la nouvelle loi sur l'instruction publique dispense désormais les pêcheurs de prendre un mousse lorsqu'ils s'inscrivent sur les rôles d'armement. Ainsi, les patrons s'en dispensent ou engagent pour cet office des hommes plus vieux et plus expérimentés. Il en résulte une gêne financière supplémentaire pour les familles de pêcheurs. Cette situation est aggravée par le fait que le revenu de ces familles est déjà grevé par la diminution du salariat féminin au sein des entreprises de conserverie, dans lesquelles travaillent femmes et filles de marins. À ces conséquences matérielles s'ajoute une autre répercussion : en raison de la diminution de l'embauche des mousses, la filiation professionnelle n'est plus systématiquement assurée, bien que ce phénomène reste d'une importance tout de même limitée ${ }^{13}$.

\section{La restriction des frais de fonctionnement}

Une des opérations les plus onéreuses est celle qui consiste à épandre la rogue afin d'appâter la sardine. La rogue ${ }^{14}$, importée de Scandinavie, est en effet une marchandise fort coûteuse, d'autant plus que le marché est aux mains de négociants qui en font un produit de spéculation dont le prix s'accroît régulièrement. Certains notables locaux sont impliqués dans cette spéculation, ce qui rend difficile une éventuelle intervention de l'État en faveur des patrons. Dans les ports où le système de partage des parts inclut également l'achat en commun de la rogue par le patron et l'équipage, comme à Port-Louis, les matelots sont aussi pénalisés. Les patrons se réunissent donc au sein de coopératives d'achat, ce qui leur permet d'obtenir des prix de revient plus avantageux et de bénéficier de la prime gouvernementale $^{15}$. Cependant, malgré le succès de ces initiatives, certains pêcheurs ne peuvent en profiter, car ils se trouvent dans une position de

12. Arch. dép. du Morbihan, M 4517 : Crises sardinières, 1908-1918, rapports de gendarmerie.

13. Ainsi la proportion de mousses parmi les équipages de Port-Louis s'élève-t-elle encore à 6,9 \% en 1900 (SHM Lorient, 9 P 4/1-4), alors que la même année elle a chutée de $48 \%$ à Palais par rapport à 1868 .

14. Rogue : œufs de morue saumurés, qui constituent le principal appât utilisé au $\mathrm{XIX}^{\mathrm{e}}$ siècle dans la pêche sardinière.

15. Cette prime gouvernementale s'élève à 15 francs par quintal de rogue acheté, ce qui peut représenter de 17 à $24 \%$ du prix de revient total. 
dépendance vis à vis des fournisseurs traditionnels. Ceux-ci leur ont en effet consenti des crédits. Jusqu'alors cependant, les négociants limitaient leur pression financière sur les pêcheurs, puisque ces derniers constituaient souvent leurs propres équipages (beaucoup de négociants étaient également armateurs). Or, les négociants se désengagent des processus d'armement. De plus, ils sont contraints d'augmenter le prix de la rogue pour compenser leurs pertes de revenus. En effet, du fait de la crise sardinière, le volume du marché de cet appât s'est restreint (il y a moins de pêcheurs sardiniers, qui utilisent donc moins de rogue). Cette évolution du cours de la rogue entraîne une augmentation conséquente des prix de revient du poisson, sans que cet effet soit compensé par une hausse des cours d'achat pratiqués par les conserveries.

Une autre solution retenue par les patrons consiste à employer des rogues artificielles ou des mélanges dans lesquels la rogue traditionnelle n'entre que dans une faible proportion. Divers succédanés ont été imaginés par des pêcheurs, mais aussi par des scientifiques ou des industriels. Cependant, l'emploi de ces types d'ersatz restera souvent au stade expérimental ou sera relativement réduit, malgré des incitations du gouvernement. Des commissions locales pour l'étude de l'emploi de rogue artificielle se sont en effet créées sous l'impulsion de l'État. De la même façon, l'intervention des scientifiques en faveur de ce type de produit, en particulier celle de l'inspecteur des pêches Paul Fabre-Domergue, membre du laboratoire de biologie maritime de Concarneau, restera presque lettre morte. Dans la continuité de recherches d'autres scientifiques tels Pouchet ou Vaillant, il tente de réhabiliter l'usage des sennes et de promouvoir les substituts de rogue, mais il restera incompris des professionnels. Malgré tout, un mélange à base d'appât traditionnel et de farine d'arachide est couramment utilisé. Il permet une réduction de frais pouvant atteindre $60 \%$ du prix de la rogue naturelle. Mais pêcheurs et négociants sont divisés quant à l'emploi de ce mélange, suspecté de diminuer la qualité des prises en réduisant la durée de conservation du poisson frais. Lors de la consultation gouvernementale de 1913, la majorité des pêcheurs morbihannais rendent à ce propos un avis défavorable ${ }^{16}$.

Une autre solution à ce problème pourrait consister à ne plus utiliser de rogue scandinave, mais de promouvoir une fabrication nationale. Les rogues de fabrication française, en effet, n'entrent que pour une très faible part dans la consommation, ceci en dépit de la prime de 20 francs par quintal accordée par le gouvernement aux terre-neuviens de Saint-Malo ${ }^{17}$. En effet, les Français pratiquent la pêche morutière durant la saison estivale, et n'ont pas la possibilité de traiter le produit à bord ou à proximité. En outre, ils ne disposent pas d'un réel savoir-faire, contrairement aux scan-

16. SAINT-Leon, Martin et De SEILHAC, Léon, La crise sardinière, Paris, Rousseau, 1913.

17. OuzzLLE, Georges, Contribution à l'étude de la crise sardinière, Lorient, Le Beau, 1909; SHM, 6 P 1/2-8, Dépêches ministérielles et correspondance à l'arrivée, quartier d'Auray, 1896-1906. 
dinaves, qui pêchent durant la saison estivale, lorsque les œufs parviennent à une maturité appropriée à la préparation. Ceux-ci disposent également de la possibilité d'effectuer quotidiennement les opérations de préparation de la rogue, du fait de la proximité entre littoraux et lieux de pêche, ce qui confère au produit une qualité nettement supérieure à celle obtenue par les marins français sur les côtes américaines. La rogue continue donc, malgré la crise, d'être importée essentiellement de Norvège, et en particulier d'Aalesund, de Christansund, et surtout de Bergen. Une faible proportion provient également de Reikjavik.

La rogue de maquereau, dont l'efficacité est reconnue par les professionnels, pourrait aussi jouer un rôle palliatif. Elle est fabriquée localement dans certains ports de la Manche ou de Haute-Normandie. Cependant, en raison d'une production restreinte sur laquelle les spéculateurs ont rapidement ma mainmise, la rogue de maquereau s'avère autant, sinon plus onéreuse, que l'appât traditionnel. Les pêcheurs restent donc fortement dépendants des négociants en rogue. L'utilisation de cet appât grève de façon importante leurs frais de production, et accroît les effets négatifs des crises sur leurs revenus.

\section{Des mutations techniques contraintes?}

\section{Les modifications du matériel de pêche}

La typologie des embarcations sardinières se diversifie. Cette évolution se double d'une diminution dans la fréquence de renouvellement des embarcations. Le bateau utilisé doit désormais pouvoir concilier plusieurs contraintes : il doit garder ses qualités de manœuvre tout en jouissant d'un prix de revient restreint. D'autre part, il doit pouvoir parvenir aussi rapidement que possible à l'usine, afin de bénéficier des meilleurs prix de vente. La situation s'inscrit en effet dans une logique de crise de débouchés : lors des périodes d'abondance du poisson, l'offre est supérieure à la demande. Les derniers équipages à se présenter aux commises d'usines bénéficient donc d'un prix de vente très largement inférieur à celui proposé aux équipages précédents. D'où l'emploi de plus en plus fréquent dès les années 1880-90 de canots, petits sloops ou "Quimperlé " de taille réduite, qui supplantent rapidement la traditionnelle chaloupe ${ }^{18}$, jugée trop lourde et difficile à manier. Ces embarcations, plus légères, plus maniables, plus effilées, ont en outre l'avantage de bénéficier d'un coût de revient réduit. Leur prix à l'achat est en effet toujours inférieur au millier de francs, alors qu'une

18. SHM Lorient, séries diverses, registres des rôles de bord au désarmement, 18501913 : la proportion de canots et petits sloops, qui était en moyenne de 0 à $8 \%$ dans les ports sardiniers morbihannais dans les années 1850-1860, atteint dans les années 19001910 une importance de 45 à 70 \% de la flottille. Cette évolution s'accélère à l'occasion de la seconde crise sardinière. Ainsi à Étel la proportion de canots augmente-t-elle de $57 \%$ entre 1901 et 1910, alors que dans le même temps la proportion de sloops connaît une évolution positive de $140 \%$. 
chaloupe traditionnelle non pontée peut être estimée à 1000 à 2000 francs environ. Il faut aussi ajouter aux frais d'amortissement des frais d'entretien annuels de la coque et du gréement, estimés à une centaine de francs. Les canots sont également plus rapides : leur vélocité est accrue par l'utilisation d'un gréement plus important. Enfin, en raison de leurs dimensions restreintes, ces embarcations ne nécessitent qu'un équipage réduit pour effectuer les manœuvres. De manière mathématique, cette réduction des effectifs de matelots entraîne une augmentation de la proportion de patrons parmi les équipages. Un canot, en effet, ne nécessite pour les opérations de pêche que une ou deux personnes, bien que cet effectif puisse également être supérieur, alors que quatre ou cinq hommes étaient indispensables à la manœuvre d'une chaloupe. En revanche, ce tonnage réduit restreint le rayon d'action de ce type d'embarcation.

L'utilisation des annexes va permettre de pallier cette difficulté tout en conservant les caractères de maniabilité propres à ces petites embarcations. Cette technique, qui se diffuse tout d'abord en Vendée et en Charente $^{19}$, permet aux armateurs de disposer d'une autre option d'évolution technologique. La chaloupe traditionnelle est réduite au rôle de bateau transporteur ou remorqueur d'embarcations plus petites, les annexes, à bord desquelles les opérations de pêche sont facilitées. L'utilisation des chaloupes permet d'atteindre des zones de pêche plus distantes que celles qu'autorise l'emploi des canots. Une fois parvenues sur les lieux de pêche, les annexes sont mises à l'eau. Dans le Morbihan, cette mutation commence à se produire dès les premières années du vingtième siècle ${ }^{20}$. Des annexes sont par exemple mentionnées dans les archives à Étel dès $1900^{21}$; mais ces sources présentent des lacunes et il ne faut pas écarter la possibilité d'une apparition antérieure de ce type d'embarcation. À l'origine, plusieurs équipages se regroupent pour pratiquer la pêche ensemble. Une chaloupe transporteuse effectue des rotations entre les zones de pêche et les usines, ce qui permet de pratiquer une pêche continue tout au long de la journée. Mais cette dernière technique sera abandonnée en raison d'une situation concurrentielle de plus en plus accentuée entre les équipages, en plus de débouchés de plus en plus réduits. L'augmentation des tonnages pêchés n'est donc plus une bonne solution. En effet, les pêcheurs morbihannais sont désormais soucieux d'éviter toute surproduction, qui serait susceptible d'engendrer une diminution des prix du mille de sardines.

19. Archives municipales de Palais, non classées : l'utilisation du canot était également répandue avant la crise dans le petit port sardinier de Sauzon en raison du faible tirant d'eau de ce port, conséquence de son envasement, et ne permettant pas d'utilisation d'embarcation de plus fort tonnage.

20. SHM Lorient, séries diverses, registres des rôles de bord au désarmement, 1910-1913 : les chaloupes annexées constituent semble-t-il 15 à $25 \%$ des embarcations morbihannaises à la veille de la Grande Guerre. Cependant, une estimation au niveau de l'arrondissement est rendue délicate de l'irrégularité des informations fournies par les sources, essentiellement des témoignages contemporains et surtout les registres de rôles de bord.

21. SHM Lorient, 6 P 6/6, registre des rôles de bord au désarmement, Étel, 1900 
Enfin, un troisième choix technologique peut consister à contrario à employer des embarcations de plus fort tonnage. Celles-ci permettent en effet de bénéficier d'un rayon d'action plus étendu et de répondre à la théorie du " plus au large " prônée par certains scientifiques, professionnels, ou hommes politiques. La traditionnelle chaloupe non pontée, en effet, adaptée à la pêche en baie (Quiberon) ou dans les courreaux, ne permet pas forcément les sorties d'hiver ou les campagnes plus au large. Mais ces embarcations plus fortes (chaloupes pontées, sloops de fort tonnage ${ }^{22}$ ) sont surtout employées en raison de leur polyvalence potentielle. Elles permettent en effet de se livrer à d'autres types de pêche (caseyage, drague, chalut) tout en conservant une activité sardinière saisonnière. Dans certains ports, comme celui de Palais, les infrastructures portuaires et surtout les systèmes de financement ne permettent pas cette évolution.

Cependant, les petits patrons-armateurs ne disposent pas toujours de la possibilité d'opérer une reconversion totale par le biais d'un changement complet d'embarcation. Cet état de fait tient à deux raisons. Les reconversions dans les autres domaines halieutiques (chalutage, mais surtout pêche thonière) nécessitent des capitaux. Leur mise en œuvre dépend souvent des choix effectués par des investisseurs locaux ou extérieurs. Si à Groix ou à Étel, certains négociants font ce choix, ce n'est pas le cas à Belle-lle ou à Riantec. Or, les patrons indépendants ne disposent pas des capitaux nécessaires à cette reconversion. La seconde raison tient aux orientations prises avant la crise sardinière. Ainsi, à Groix, le virage de la pêche thonière est-il amorcé dès les années 1870, grâce à des réinvestissements de bénéfices et au concours des négociants locaux. À Étel, à Quiberon, comme au Croisic ou à La Turballe, certains pêcheurs profitent des subventions gouvernementales allouées sur le budget de la Marine ${ }^{23}$ pour faire construire des unités destinées au chalutage. Mais dans d'autres ports comme à BelleÎle, cette orientation est prise tardivement, au moment même ou le gouvernement remet en question l'attribution de ces allocations, dans les années 1876-1877.

Cette politique gouvernementale d'attribution de subventions, si elle joue un rôle important dans le choix d'une orientation vers des pêches alternatives, n'est cependant pas un facteur essentiel de décision. Toutefois, elle encourage les initiatives individuelles, dans la mesure où certains patrons ou armateurs peuvent également bénéficier de capitaux d'origine privée. Cette situation, évoquée précédemment dans certains ports en ce qui concerne le chalutage, se vérifiera également pour les campagnes de la morue verte. Celles-ci sont encouragées par un système de primes prévues par la loi du 26 février 1911 et par le décret d'encouragement aux gran-

22. SHM Lorient, 9 P 4/28-30 : on peut également relever certains exemples de dundees armés à la part au thon ou aux pêches d'hiver, mais aussi à la sardine, comme à PortLouis en 1909.

23. SHM Lorient 6 P 1/1, Correspondance et dépêches ministérielles, quartiers maritimes d'Auray et de Belle-lle, 1867-1877. 
des pêches maritimes du 9 novembre 1911. Malgré tout, c'est l'initiative prise à l'origine par deux armateurs locaux, un portlouisien et un grésillon, grâce à des capitaux privés, et avec l'aide de ces subventions, qui sera décisive. La tentative, qui se développe par le biais de la fondation d'un syndicat coopératif breton-mauritanien en septembre $1912^{24}$, ne dépassera pourtant guère le stade expérimental au niveau départemental. En raison d'un certain manque d'expérience en ce domaine halieutique particulier, mais aussi à cause d'un manque de réussite certain lors des premières campagnes, seules quelques unités de Groix, d'Étel ou de Port-Louis s'engageront pour quelques saisons à Port-Étienne. Les problèmes liés aux reconversions halieutiques vont donc au-delà des évolutions des embarcations proprement dîtes : elles dépendent surtout de la possibilité de disposer de capitaux d'origine privée.

Reste cependant à faire mention de la révolution de la pêche motorisée. Dans le domaine de la pêche sardinière, la motorisation va rester très longtemps l'apanage des pinasses d'Arcachon et de quelques embarcations de Saint-Jean-de-Luz. Le moteur est adapté sur les pinasses de ce dernier port dès 1906, comme l'ont déjà fait les pêcheurs espagnols. Dès cette époque débutent également les essais d'adaptation du moteur à explosion sur les pinasses d'Arcachon, avec des résultats probants. La motorisation permet en particulier aux patrons de traînières de disposer d'un rayon d'action plus étendu et de revenir plus rapidement au port. Mais elle ne concernera la Bretagne qu'à partir des années $1920^{25}$. Les pêcheurs bretons, peu enclins à la modernité, se méfient de cette innovation. En outre, les tentatives d'adaptation d'un moteur sur les lourdes chaloupes sardinières ne sont guère concluantes. Les chaloupes bretonnes, trop pesantes, nécessitent trop d'essence pour un gain de vélocité modeste. Il faudra donc attendre la fin des années 1900 pour voir apparaître en Bretagne un modèle hybride, dérivé à la fois de la chaloupe bretonne et de la pinasse arcachonaise. Mais les sardiniers motorisés vont être très lents à s'imposer en Bretagne : en 1911, seules trois unités y sont en activité. Une douzaine d'années plus tard, le nombre de sardiniers motorisés n'y aura guère que décuplé, et la voile sera encore la règle, contrairement aux pêcheurs sardiniers marseillais qui durant la même période auront adopté un moteur sur plus de 600 " pointus ", dont les conditions de navigation sont certes moins délicates.

\section{L'évolution des engins de pêche}

Les filets de coton issus des filatures mécaniques apparaissent dans les années 1880. Ces filets droits, composés de mailles beaucoup plus fines que les traditionnels filets de chanvre, sont donc plus discrets et réputés plus "pêchants ». Désormais commandés à Paris, ils suscitent quelques

24. Arch. dép. du Morbihan, 10 M 102, Syndicats professionnels.

25. Bien que certains auteurs affirment l'existence d'embarcations motorisées à Quiberon et Port-Louis, ce que ne confirme aucune des sources consultées. 
critiques parmi les pêcheurs. Celles-ci tiennent en premier lieu à leur coût, de 80 à 100 francs ${ }^{26}$ pièce, alors même que marins et patrons se trouvent en situation financière instable. Or, chaque équipage, pour être bien équipé, doit embarquer un ou deux jeux de 15 filets droits, de différents moules ${ }^{27}$ pour faire face aux différentes catégories de sardines. L'utilisation de ce type de matériel implique des frais d'amortissement et d'entretien très élevés (2000 à 3000 francs annuellement), d'autant que ces filets sont beaucoup moins résistants que les filets traditionnels et doivent donc être renouvelés beaucoup plus fréquemment, tous les trois ou quatre années. En outre, l'utilisation de ces nouveaux engins peut entraîner un manque à gagner dans les familles de pêcheurs, ou la tâche de confection des filets droits revenait traditionnellement à des femmes, veuves, ou enfants de pêcheurs.

Les filets sont également au cœur de dissensions entre les pêcheurs de la côte atlantique, en raison de l'utilisation par certains d'entre-eux de nouveaux types de filets pour la pêche à la sardine. Le filet tournant, ou senne Guézennec, est l'engin qui provoqua le plus d'hostilités. Contrairement au filet droit, passif et ou la sardine venait se prendre par les ouïes dans les mailles à la suite de l'opération consistant à appâter, la senne était un engin actif qui permettait de capturer le poisson en l'encerclant. Elle permet de capturer quotidiennement jusqu'à 150000 poissons, soit un résultat très largement supérieur à celui obtenu avec les filets droits. L'usage des sennes n'était pas nouveau. La Revue Maritime abordait déjà le sujet en 1864 :

"Tout dernièrement, des essais ont été pratiqués pour l'usage d'un filet, dont le nom breton (ar boul grunn) veut dire économisateur [sic] de rogue. $\mathrm{Au}$ lieu de prendre la sardine avec des filets ou elle se maille, on l'enveloppe dans une seine manœuvrée par deux bateaux de pêche $[\ldots]^{28}$."

Des pêcheurs l'avaient employé avec succès, notamment dans le Finistère et plus particulièrement à Audierne, où les équipages qui avaient utilisé les sennes avaient rapporté aux usines des quantités considérables de poisson. Or, cet afflux massif avait entraîné une chute des cours du poisson. En outre, un certain esprit corporatiste, un rejet de la modernité, et des jalousies certaines entre marins de différentes localités avaient incité certains pêcheurs à prendre position contre l'utilisation de ce type d'engin. À la suite de plaintes de certains d'entre eux auprès de l'administration maritime, l'utilisation des sennes avait donc été restreinte, puis interdite sur l'ensemble du littoral breton. De plus, l'engin était accusé de détruire les fonds marins et de menacer les écosystèmes en ne distinguant ni les espèces pêchées, ni la taille du poisson. En outre, la masse du pois-

26. À titre de comparaison, le gain moyen d'un pêcheur sardinier est en 1895, année de pêche sardinière jugée " moyenne " (Arch. dép. du Morbihan, 1 Z 175, Situations industrielles), de 200 à 420 francs (ministère de la Marine, Statistique des pêches maritimes, Paris, Imprimerie nationale, 1896).

27. Espace d'écartement entre les mailles.

28. La Revue Maritime, t. XII, 1864, p. 264. 
son écrasait à l'intérieur des filets une partie des sardines et les écaillait, en diminuant la valeur marchande. En 1888, puis en 1912, le gouvernement avait confirmé, sous la pression des syndicats, l'interdiction de ces engins de pêche. D'ailleurs, comme le rappellera ultérieurement le ministre de la Marine Marchande Léon Meyer en 1932, le gouvernement saurait-il engager les marins à perfectionner leur mode de pêche avant que les usiniers n'aient procédé à une amélioration de leur matériel et de leurs méthodes de production susceptibles d'accroître les capacités d'absorption de leurs entreprises? Les évolutions techniques des matériels de pêche ne sont ainsi envisageables que si elles sont en adéquation avec les débouchés de cette activité : il ne sert à rien de produire davantage de poisson si celui-ci ne peut être traité.

Pourtant, des essais menés au large de Belle-Île en 1908 s'étaient avérés concluants : les prises avaient été bonnes alors que la sardine ne " maillait " pas sur les filets droits. Il était en effet fréquent en période de crise que des bancs nombreux fussent signalés alors que le résultat de la pêche s'avérait globalement médiocre. De plus, l'utilisation de la senne (malgré l'interdiction) dans certains ports du Finistère ou en Espagne avait permis aux équipages locaux de retrouver une certaine prospérité. À Groix, le tonnage important des navires avait permis aux pêcheurs de prospecter au large, et donc de s'affranchir de la limite des trois milles (en deçà de laquelle la pêche aux sennes était en théorie interdite). Il restait le seul port morbihannais à utiliser ce type d'engin, contrairement à toutes les autres localités de l'arrondissement où la sardine était prise au filet droit ${ }^{29}$. Et grâce à un rendement trois fois plus élevé qu'ailleurs dans le département, le gain brut par homme d'équipage y était également beaucoup plus élevé. Malgré ce volume des prises élevé, le prix moyen du poisson était même légèrement plus élevé que sur le reste du littoral. Cependant seules six unités œuvraient en 1910 à la sardine à Groix, où l'activité halieutique était essentiellement thonière. Le volume pêché était donc suffisamment limité pour éviter un sur-approvisionnement des usines de l'île. Ce modèle particulier n'était donc pas applicable à l'ensemble des ports.

En fait, deux logiques s'affrontent. L'une d'elle consiste à vouloir augmenter les revenus des prises en augmentant de manière massive la production, quitte à entraîner une diminution des prix d'achat, qui serait compensée par l'augmentation des volumes. La seconde logique consiste à stabiliser la production afin de stabiliser les cours. Les pêcheurs ont en effet dans leur grande majorité des difficultés à saisir les mécanismes qui régissent un marché désormais international et soumis aux lois de l'offre et de la demande. Avant la crise, lorsque la France détenait le quasi-monopole de la production de sardine en conserve, toutes les sardines pêchées étaient achetées par les usines à des cours ne subissant que de très faibles variations. Désormais, les cours à l'achat restent élevés en cas de pêche médiocre. Mais toute période d'abondance entraîne une chute des prix. Les filets

29. Statistiques des pêches maritimes pour l'année 1910. 
tournants, dans cette logique, sont accusés de pouvoir causer une surproduction, donc une chute des cours, et par conséquent des revenus. Les fabricants, quant à eux, pensent que l'utilisation de ces engins est le seul moyen de mener des campagnes efficaces et de sauver la production nationale.

Cette divergence de vue suscite un certain nombre de tensions et de conflits, qui incite la Fédération Syndicale des Pêcheurs Sardiniers de France à organiser à la fin de l'hiver 1913 un vote dans les centres sardiniers au sujet du bien-fondé de l'utilisation de la senne. Dans le Morbihan comme dans toute la Bretagne ${ }^{30}$, à l'exception de certains ports finistériens, la très grande majorité des marins consultés se prononcent contre l'utilisation de l'engin. À Belle-Île par exemple, 97,7 \% des votants s'y déclarent opposés, le reste des suffrages étant constitué de bulletins blancs. Dans certains ports, les marins qui ont osé revendiquer le droit d'utiliser la senne sont mis à l'écart de la communauté. Dans d'autres localités sardinières, les marins se prononcent contre cette utilisation, mais sont prêts à renoncer aux limitations des prises si les usiniers s'engagent à traiter la totalité du poisson pêché et à garantir un prix minimum d'achat. Mais si certains marins sont disposés à ces concessions, comme à Étel (mais aussi à BelleÎle, ou dans le Finistère à Douarnenez et Concarneau), beaucoup de pêcheurs et de fabricants reconnaissent qu'en cas de pêche abondante les entreprises n'auront pas la capacité d'absorber toutes les prises. Une commission d'arbitrage mixte comprenant pêcheurs et fabricants parvient néanmoins après de longs mois de blocages et de négociations à conclure un accord. En vertu de celui-ci, les filets coulissants pourront être utilisés de manière exceptionnelle durant la campagne estivale 1913, sous réserve que la pêche soit peu abondante et que les pêcheurs ne puissent parvenir à fournir les deux-tiers de la demande des usines à l'aide des filets droits conventionnels. Les sennes sont autorisées dans une proportion de un filet pour cinquante bateaux. En échange de quoi un prix minimum d'achat est garanti par les fabriquants. Mais malgré une saison très médiocre et des volumes de pêche très réduits, les termes de cet accord, qui porte également sur la non-utilisation de la rogue artificielle, ne seront pas respectés par les pêcheurs, qui sauf exception refuseront l'emploi du filet tournant. La révolution du filet tournant sera très longue à s'imposer, comme en témoignera l'inspecteur principal de l'OSTPM encore en 1948 : « La supériorité de la senne tournante sur le filet droit est incontestable, mais la lutte sera longue pour la faire accepter. "

Toujours dans la même logique, les pêcheurs morbihannais se prononcent également en faveur de l'usage d'un unique canot annexe par chaloupe $^{31}$. Cette décision est prise par crainte de la surproduction. Pourtant,

30. L'ouvrage de Martin Saint-Léon et Léon de Seilhac, op. cit., p. 48-53 donne les résultats de cette consultation, que André Marie D'Avigneau, op. cit., évoque également p. 249, tout comme divers journaux régionaux contemporains.

31. Amieux, Louis, Documents concernant la crise sardinière de 1903 à 1912, Nantes, Dugas, 1919, p. 88 et journal Le Phare de la Loire, 17 avril 1911. 
l'utilisation de deux ou trois annexes par embarcation a permis de rétablir la prospérité dans le port sardinier des Sables d'Olonne. Les syndicats interdiront aussi la pêche le dimanche - mesure pour laquelle ils seront soutenus par les prêtres - et la limiteront durant la semaine à une seule sortie quotidienne, alors qu'il était fréquent lors des saisons estivales précédant la crise d'effectuer deux sorties, le matin et en début d'après-midi.

Ainsi les pêcheurs confrontés à un marché de plus en plus ouvert sontils quelquefois hésitants face à une modernisation qui bouleverse parfois leurs techniques et leurs concepts économiques traditionnels. Si les pêcheurs morbihannais font preuve de capacités d'adaptation à la crise, s'ils participent à la recherche de solutions, ils ne prennent pourtant que peu d'initiatives. Celles-ci sont plutôt le fait des armateurs extérieurs, des entreprises ou des structures gouvernementales. L'impulsion ou l'aide de l'État reste souvent nécessaire. Quant aux innovations adoptées par les pêcheurs locaux, elles ne sont souvent que les reflets d'initiatives extrarégionales qui ont fait la preuve de leur efficacité. En effet, les difficultés de compréhension des mécanismes de la crise suscitent une certaine inertie, aggravée par un esprit corporatiste et un espoir de retour à une certain " âge d'or " de l'activité sardinière. Malgré tout, la crise engendre une seconde révolution technologique dans le domaine des pêches sardinières, après celle issue quelques dizaines d'années plus tôt de la transition des presses aux conserveries, et de moindre importance.

\section{Bibliographie}

Dubors, Xavier, «Mutations techniques et crises sardinières ", Actes du colloque de Lorient, 1999, Mutations techniques des pêches maritimes, Plouzané, Éditions IFREMER, 2001, p. 41-50.

DuPouY, Auguste, Pêcheurs bretons, Paris, Boccard, 1920, 136 p.

LE GALL, Théodore, L'industrie de la pêche dans les ports sardiniers bretons : crises et palliatifs, thèse dactylographiée de droit-économie, Université de Rennes, 1904, 250 p.

LEGENDRE, M., "La rogue, son histoire, son remplacement ", Revue des travaux de l'Office Scientifique et Technique des Pêches Maritimes, $n^{\circ}$ 13, 1944, p. 591-597

Mollat, Michel, Histoire des pêches maritimes en France, Toulouse, Privat, 1987, $407 \mathrm{p}$.

MORDREL, Louis, Les institutions de la pêche maritime, histoire et évolution. Essai d'interprétation sociologique, Thèse dactylographiée d'économie et sciences sociales, Université de Paris II, 1972, 848 p.

Ouzille, Georges, Contribution à l'étude de la crise sardinière, Lorient, Le Beau, 1909, $164 \mathrm{p}$.

SAINT-LEON, Martin et DE SEILHAC, Léon, La crise sardinière, Paris, Rousseau, 1913, $204 \mathrm{p}$.

SEILHAC, Léon DE, Marins-pêcheurs : pêcheurs côtiers et pêcheurs de morue à Terre Neuve et Islande, Paris, Rousseau-Challamel, 1899, 199 p. 
TOSELlo-BANCAL, François, L'évolution de la pêche de la sardine sur le littoral français, Thèse dactylographiée, Université de Paris IV-Sorbonne, 1994, 216 p.

VOURCH, François, Analyse des rapports sociaux dans la pêche artisanale, histoire et évolution, Paris, éditions CORDES, XIV-159 p.

\section{RESUME}

La crise sardinière va imposer aux pêcheurs morbihannais un certain nombre de contraintes nouvelles, dont ils vont devoir tenir compte pour assurer la pérennité de leurs activités professionnelles. Les principaux acteurs de cette économie halieutique sont dès lors confrontés à un certain nombre de choix. En effet, les armateurs traditionnels, qui jusqu'alors assumaient à eux seuls la plus grande partie du pouvoir décisionnel, se désengagent de l'activité. Les pêcheurs eux-mêmes, qui ne peuvent pas toujours s'engager dans la voie de la reconversion - qui dépend des capacités d'évolution des modes de financement et d'armement - vont donc devoir adopter ou subir certaines évolutions structurelles et techniques pour s'accommoder de la crise. Mais les capacités d'adaptabilité des marins morbihannais, qui peuvent également dépendre de facteurs extérieurs, se heurtent à la rigidité des méthodes de travail traditionnelles et des mentalités, issue en partie d'une incompréhension face aux mécanismes de la crise.

\section{ABSTRACT}

The crisis of the french sardine fishing lays dows to the South Brittany fishermen some new constraints, which they have to take into consideration to assure the perenniality of their professionnal activities. The actors of this halieutic economy, from that time onwards, are compelled to do some choices. Indeed, the traditional ship-owners - who assume until then the decisions abilities in most cases- take their activities out of pawn. The fishermen, who may not ever turn over to other employments, will so have to carry or to submit to some structural or technical evolutions to put up to the crisis. But the South Brittany seamen capacities of adaptability - which may depends on external factors - have to come up against the inflexibility of the mental habits and the traditional methods of working, partly resulting from a lack of understanding in the crisis gear. 